\title{
FLUORESCÊNCIA DA CLOROFILA $a$ EM PLANTAS DE PESSEGUEIRO DA CULTIVAR MACIEL ENXERTADA SOBRE DIFERENTES PORTA-ENXERTOS ${ }^{1}$
}

\author{
EMANUELA GARBIN MARTINAZZO², DIOLINA MOURA SILVA ${ }^{3}$, \\ VALMOR JOÃO BIANCHI ${ }^{4}$, MARCOS ANTONIO BACARIN ${ }^{4}$
}

RESUMO - O objetivo deste trabalho foi avaliar características da fluorescência da clorofila $a$ em plantas de pessegueiro da cv. Maciel enxertadas em seis porta-enxertos. As análises da fluorescência da clorofila $a$, do índice de clorofila e do comprimento das folhas iniciaram-se após o período invernal e foram repetidas até aproximadamente 150 dias, totalizando seis coletas. O início da ontogenia das folhas foi caracterizado por alguns parâmetros de fluorescência mais elevados. Elevada pluviosidade, ocorrida entre os dias $61 \mathrm{e}$ 112 do início do experimento, foi fator ambiental que causou maior alteração nos parâmetros do Teste JIP, principalmente aqueles relacionados ao fotossistema I. O índice de clorofila não foi influenciado pelo efeito das associações copa $x$ porta-enxerto. Queda nos valores de $\mathrm{PI}_{\text {TOTAL }}$ foram verificadas no período de maior precipitação, comportamento que foi independente dos porta-enxertos, exceto para as plantas enxertadas no porta-enxerto 'Rubirá', que apresentaram menores danos ao aparato fotossintético. De maneira geral, os porta-enxertos estudados, em combinação com a cv. Maciel, não afetaram a absorção e o aproveitamento da energia luminosa pelo sistema fotossintético.

Termos para indexação: crescimento foliar, Teste JIP, compatibilidade.

\section{CHLOROPHYLL $a$ FLUORESCENCE IN PEACH CULTIVAR MACIEL GRAFTED ON DIFFERENT ROOTSTOCKS}

\begin{abstract}
The objective of this study was to evaluate characteristics of chlorophyll fluorescence in peach trees cv. Maciel grafted on six rootstocks. The analysis of the chlorophyll $a$ fluorescence, the chlorophyll content and length of the leaves began after the winter period and were repeated until approximately 150 days, totaling six samples. The early ontogeny of the leaves was characterized by some higher fluorescence parameters. High rainfall, which occurred between 61 and 112 days of the beginning of the experiment, was the environmental factor that caused the greatest change in the parameters of the JIP Test, especially those related to photosystem I. The chlorophyll content was not influenced by the effect of associations cup $x$ rootstock. Fall in the $\mathrm{PI}_{\text {TOTAL }}$ were in the period of highest rainfall, behavior that was independent of the rootstock, except for plants grafted on rootstock Rubira, who had minor damage to the photosynthetic apparatus. In general, rootstocks studied in combination with cv. Maciel did not affect the absorption and utilization of light energy by photosynthetic system.
\end{abstract}

Index terms: leaf growth, JIP Test, compatibility.

${ }^{1}$ (Trabalho 234-11). Recebido em: 30-09-2011. Aceito para publicação em: 10-08-2012

${ }^{2}$ Bióloga. Doutora em Fisiologia Vegetal - Departamento de Botânica - Instituto de Biologia - Universidade Federal de Pelotas - RS, Câmpus Capão do Leão, C. P. 354 - 96010-900 E mail: martinazzo@gmail.com

${ }^{3}$ Prof. Doutora do Centro de Ciências Humanas e Naturais - Departamento de Ciências Biológicas - Universidade Federal do Espírito Santo - ES, Câmpus Universitário, Avenida Fernando Ferrari, 514, Goiabeiras-ES, 29075-910. E mail: diu@terra.com.br

${ }^{4}$ Prof. Doutor do Dep ${ }^{\text {to }}$ de Botânica - Instituto de Biologia - Universidade Federal de Pelotas - RS, Câmpus Capão do Leão, C. P. 354 96010-900. E mails: valmorjb@yahoo.com; marcos.baccarin@gmail.com 


\section{INTRODUÇÃO}

O Rio Grande do Sul é o principal Estado brasileiro em área de cultivo de rosáceas, respondendo por mais de $50 \%$ da produção nacional. O sucesso do cultivo do pessegueiro na região sul do Estado depende da utilização de cultivares-copa cujos frutos atendam à demanda do mercado, adaptadas às condições de inverno, e mudas de qualidade, garantindo a alta produtividade do pomar (HERTER, 2002).

Plantas cultivadas em campo são, frequentemente, expostas a condições ambientais adversas que afetam o crescimento (WANG et al., 2007). A vitalidade das plantas é dependente da capacidade de adaptação a determinadas condições ambientais, a exemplo daquelas relacionadas ao déficit hídrico, alagamento, luminosidade, entre outras, e qualquer mudança é fator de estresse que induz a perturbação do crescimento (TSIMILLI-MICHAEL;STRASSER, 2008).

A escolha do porta-enxerto a ser utilizado para determinada cultivar-copa é importante fator e deve ser considerado na produção de espécies frutíferas, por influenciar na absorção de água, nutrientes e fixação das plantas ao solo, podendo variar em função das condições edafoclimáticas locais, afetando a produtividade (GOTO et al., 2003).

Entre as ferramentas utilizadas para estudar os efeitos das mudanças ambientais sobre o aparato fotossintético destaca- se a fluorescência da clorofila $a$, que é um método simples, rápido, sensível e não destrutivo, amplamente utilizado em investigações de danos e/ou reparos causados no sistema fotossintético das plantas, em função de diversos tipos de estresse (STRASSER et al., 2004; EULLAFFROY et al., 2009).

Os dados de intensidade de fluorescência transiente (OJIP) podem ser analisados através do Teste JIP, desenvolvido por Strasser e Strasser (1995). O uso dos parâmetros calculados a partir do Teste JIP permite a quantificação da eficiência de absorção e aproveitamento da energia luminosa através da cadeia de transporte de elétrons da fotossíntese. Além de avaliar a funcionalidade do fotossistema II (FSII), reflete a taxa de transporte de elétrons no interior da membrana dos tilacoides e o subsequente funcionamento da ferredoxina-NADP oxirredutase e ciclo de Calvin (SCHANSKER et al., 2003).

O presente trabalho objetivou avaliar as características de fluorescência da clorofila $a$ em plantas de pessegueiro da cultivar Maciel, enxertadas em diferentes porta-enxertos, durante um ciclo de crescimento das plantas.

\section{MATERIAL E MÉTODOS}

O experimento foi conduzido no período compreendido entre agosto de 2009 e janeiro de 2010, no Centro Agropecuário da Palma no Município de Capão do Leão-RS, na latitude 3152'S, longitude $52^{\circ} 21^{\prime} \mathrm{W}$ e altitude média de $13 \mathrm{~m}$. O clima da região caracteriza-se por ser temperado, com chuvas bem distribuídas, e verão quente, sendo, pela classificação de Köppen, do tipo Cfa. Os dados meteorológicos foram obtidos na estação meteorológica da Embrapa Clima Temperado.

Plantas de pessegueiro com quatro anos, da cultivar Maciel, enxertadas sobre seedlings de seis porta-enxertos de Prunus persica (Indústria, Aldrighi, Tskuba, Okinawa Clone 2, Okinawa Clone 12 e Rubirá) foram utilizadas no experimento. Ressalta-se que o material identificado como Indústria refere-se a seedlings obtidos de caroços provenientes da indústria de processamento de pêssegos, sem identidade varietal. Os pomares foram implantados no ano de 2005 no espaçamento de 4,5 m x 1,0 m e sistema de condução em "Y". Os tratamentos fitossanitários seguiram as recomendações técnicas específicas para o sistema de produção integrada de pêssego.

As avaliações de fluorescência transiente OJIP das clorofilas, comprimento das folhas e índice de clorofila iniciaram-se em 27 de agosto de 2009, após o início da brotação, sendo repetidas até, aproximadamente, 150 dias do início das coletas. As folhas foram padronizadas de forma a se utilizar sempre folhas dos mesmos ramos do ano nas avaliações. O comprimento da folha foi medido com régua milimetrada, desde a inserção do pecíolo no limbo foliar até o final da nervura central. Os teores de clorofila foram estimados pelo medidor portátil de clorofila CL-01 Hansatech Instruments Ltd., King's Lynn Norfolk, UK, conforme metodologia descrita por Cassol et al. (2008), expressos pelo índice de clorofila. As mesmas folhas foram utilizadas para as medições da fluorescência da clorofila $a$.

Para a indução da fluorescência da clorofila $a$, foram utilizadas seis plantas para cada tratamento de porta-enxerto e duas medidas por planta, em lados opostos da copa, totalizando 12 repetições por tratamento. As medidas de fluorescência da clorofila foram realizadas em folhas intactas, ligadas à planta e adaptadas previamente ao escuro, por 30 minutos. A fluorescência transiente da clorofila $a$ foi medida por fluorômetro portátil (Handy-PEA, Hansatech Instruments Ltd., King's Lynn Norfolk, UK), induzida pela luz vermelha com pico de 650 $\mathrm{nm}$, de aproximadamente $3.000 \mu \mathrm{mol} \mathrm{m} \mathrm{m}^{-2} \mathrm{~s}^{-1}$. Para análise da fluorescência transiente da clorofila $a$, 
foi utilizado o Teste JIP (STRASSER et al., 2004; TSIMILLI-MICHAEL;STRASSER, 2008), o que permite calcular os fluxos específicos por centro de reação, eficiência ou rendimentos quânticos e índices de performance.

O delineamento estatístico foi inteiramente casualizado, em esquema fatorial 6 x 6 (seis combinações de porta-enxertos com a cv. Maciel e seis épocas de coleta dos dados, aos 0; 28;61; 97; 112 e 152 dias após o início do experimento - DAIE). Os dados foram submetidos à análise de variância e ao teste de Tukey, a 5\% de probabilidade de erro.

\section{RESULTADOS E DISCUSSÃO}

Os dados de temperatura e precipitação, no período experimental foram típicos para o fim da estação de inverno, toda a primavera e início do verão. Contudo, ocorreu aumento na média da precipitação no mês de novembro (dados não apresentados), em que o acumulado das chuvas foi quatro vezes superior às normais climatológicas referentes a um período de 30 anos (EMBRAPA, 2010).

No início do experimento, as folhas apresentaram os menores tamanhos (Figura 1A), porém não houve efeito da interação tipo de porta-enxerto com a cv. Maciel, sendo verificado apenas efeito do fator dias após o início do experimento. O mesmo foi observado para os índices de clorofila (Figura $1 \mathrm{~B})$, os quais decresceram até aproximadamente 60 DAIE, com posterior incremento até 112 DAIE e decréscimo ao final do experimento.

Em relação aos parâmetros de fluxo por centro de reação (RC) (Figura 2), deve ser destacado que o fluxo de absorção (ABS/RC), de captura (TR/RC), de transporte de elétrons (ET/RC) e de dissipação (DI/ $\mathrm{RC}$ ) foram influenciados apenas pelos dias após o início do experimento, apresentando comportamento similar entre as combinações de porta-enxertos e a cv. Maciel. Valores mais elevados para tais parâmetros foram observados no início do experimento (folhas mais jovens), com decréscimo aos 28 DAIE e estabilização até 112 dias, com nova elevação ao final das coletas, corroborando as afirmativas de que folhas jovens, apesar de não apresentarem aparato fotossintético totalmente desenvolvido, possuem mecanismos eficientes para absorver energia através dos complexos de captação de luz (JIANG et al., 2006)

A utilização do Teste JIP permite o entendimento dos processos associados ao fluxo energético na cadeia de transporte de elétrons da fotossíntese, expressando uma cascata de energia. Esta cascata inicia-se com a absorção da luz pelos pigmentos an- tena do FSII e termina com a redução dos aceptores finais de elétrons do fotossistema I (FSI) (YUSUF et al., 2010). As associações da cv. Maciel com os porta-enxertos relacionados não acarretaram modificações biofísicas importantes no aparato fotossintético durante o período experimental avaliado.

O fluxo de redução do aceptor final de elétrons (RE/RC) (Figura 2E) apresentou os maiores valores no início do experimento. A redução observada entre os dias 97 e 112 foi variável em função do porta-enxerto, sendo que 'Rubirá' e 'Tsukuba' foram os que induziram as menores reduções nos valores de RE/RC, aos 97 DAIE, em relação ao início do experimento, podendo sugerir que o excesso de água afetou menos estas combinações com 'Maciel' em termos de redução do aceptor final de elétrons do FSI.

Quanto ao fluxo de energia intermediário, representado pelo fluxo de transporte de elétrons além de $\mathrm{Q}_{\mathrm{A}}{ }^{-}$(ET/RC), observou- se os menores valores para a 'Maciel' sobre 'Rubirá'. Entretanto, a redução dos aceptores finais de elétrons foi o parâmetro que apresentou maior queda dos valores aos 97 DAIE, talvez devido à inativação de uma fração do RCs do FSII o que resultou em aumento do tamanho médio da antena por centro de reação ativo (YUSUF et al., 2010). A literatura tem demonstrado queda nos valores de ET/RC em várias espécies, quando submetidas ao alagamento, como plantas de canola (PERBONI et al., 2012 ), corroborando os resultados obtidos.

O rendimento quântico fotoquímico primário $\Phi_{\text {Po }_{0}}$ (Figura 3A) foi similar entre todos os tratamentos, indicando que as combinações não alteram tal parâmetro. Destaca-se que a combinação 'Rubirá' $x$ 'Maciel', mesmo apresentando menores valores de $\Phi_{\text {Eo }}$ até os 61 DAIE (Figura 3B), elevou o rendimento do transporte de elétrons no período de maior precipitação. Embora o rendimento quântico da fotossíntese possa ser alterado por fatores fotoquímicos e não fotoquímicos (STRASSER et al., 2004), verificou-se que o comportamento fotoquímico do metabolismo fotossintético foi semelhante para as combinações, não interferindo no rendimento fotoquímico da fotossíntese da copa 'Maciel'.

Em relação ao rendimento de dissipação de energia $\left(\Phi_{\text {Do }}\right)$ (Figura 3C), houve uma queda até os 97 DAIE, período posterior ao maior volume hídrico. Contudo, aos 152 DAIE observou-se aumento nos valores, com destaque para a combinação 'Rubirá' x 'Maciel'. A eficiência com que um éxciton capturado no centro de reação pode mover um elétron dentro da cadeia de transporte de elétrons de $\mathrm{Q}_{\mathrm{A}}{ }^{-}$para os aceptores finais de elétrons do FSI ( $\Phi_{\text {Ro }}$, Figura $\left.3 D\right)$, 
apresentou os menores valores aos 97 DAIE para os seis porta-enxertos. A associação 'Rubirá' x 'Maciel' foi aquela que manteve os valores muito próximos dos da primeira coleta e, aos 152 dias, alcançou o maior rendimento do transporte até os aceptores finais de elétrons do FSI.

O início do experimento foi caracterizado pela maior probabilidade do éxciton capturado mover um elétron além de $Q_{A}{ }^{-}\left(\Psi_{E o}\right)$ (Figura 3E). Após 97 DAIE, a eficiência com que um elétron pode ser movido do intersistema de aceptores de elétrons reduzidos para o aceptor final de elétrons do FSI $\left(\delta_{\mathrm{Ro}}\right)$ (Figura 3F), apresentou valores menores em comparação aos determinados aos 61 dias, coincidindo com o período de alta precipitação. Comportamento similar pode ser observado para a eficiência com que um éxciton capturado no centro de reação pode mover um elétron dentro da cadeia de transporte de elétrons de $\mathrm{Q}_{\mathrm{A}}{ }^{-}$para os aceptores finais de elétrons do FSI $\left(\rho_{o}\right)$ (Figura 3G). Deve ser destacado que a combinação 'Rubirá' x 'Maciel’ foi a que apresentou menor queda nesse período, sendo estatisticamente maior que os demais porta-enxertos associados à mesma cultivar-copa.

Os valores de $\mathrm{PI}_{\mathrm{ABS}}$ (Figura 4A) foram crescentes até os 97 dias, com posterior queda aos 112 dias e manutenção dos valores aos 152 dias, exceto para os porta-enxertos 'Indústria' e 'Rubirá', que apresentaram aumento no valor desse parâmetro, na última coleta. O índice de performance total $\left(\mathrm{PI}_{\mathrm{TOTAL}}\right)$, produto do índice de performance e a probabilidade com que um éxciton pode mover um elétron para os aceptores finais de elétrons do FSI (TSIMILLI-MICHAEL; STRASSER 2008) (Figura 4B), foi influenciado por uma queda considerável das reações de oxirredução $\left(\delta_{\mathrm{Ro}}\right)$, principalmente aos 97 dias. Esse comportamento foi independente dos porta-enxertos, exceto para as plantas enxertadas sobre 'Rubirá', que mantiveram valores de $\mathrm{PI}_{\text {TOTAL }}$ maiores que a primeira coleta durante toda a condução do estudo. Efeito similar foi observado em plantas de tomate micro-tom expostas ao alagamento, onde o parâmetro $\mathrm{PI}_{\text {TOTAL }}$ foi o mais influenciado pelo estresse (HUTHER et al., 2011).
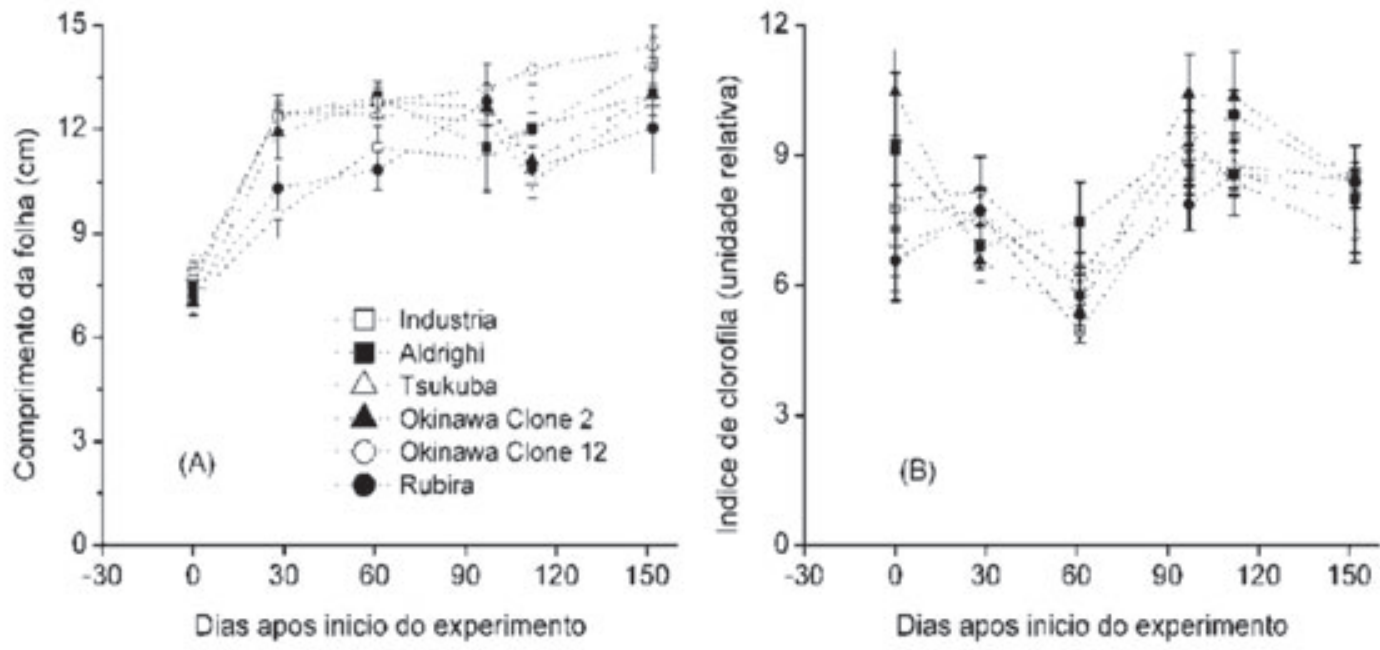

FIGURA 1- Comprimento da folha (A) e índice de clorofila (B) de plantas da cultivar Maciel enxertadas em seis porta-enxertos. 

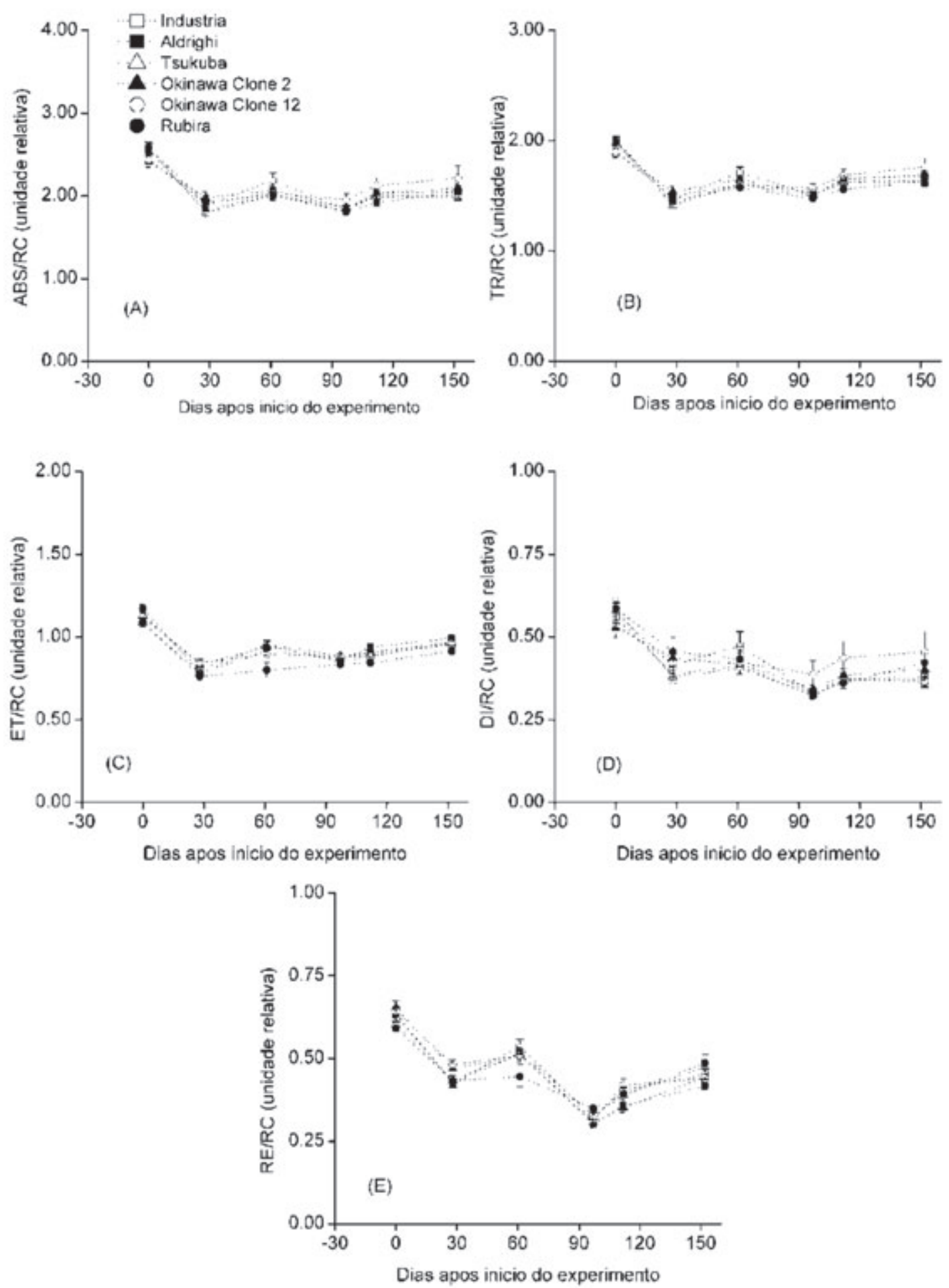

FIGURA 2 - Fluxos específicos de absorção, ABS/RC (A); de captura, TR/RC (B); de transporte de elétrons; ET/RC (C); de dissipação DI/RC (E), e de redução do aceptor final de elétrons do fotossintema I, RE/RC (E), de plantas da cultivar Maciel enxertadas em seis porta-enxertos. 

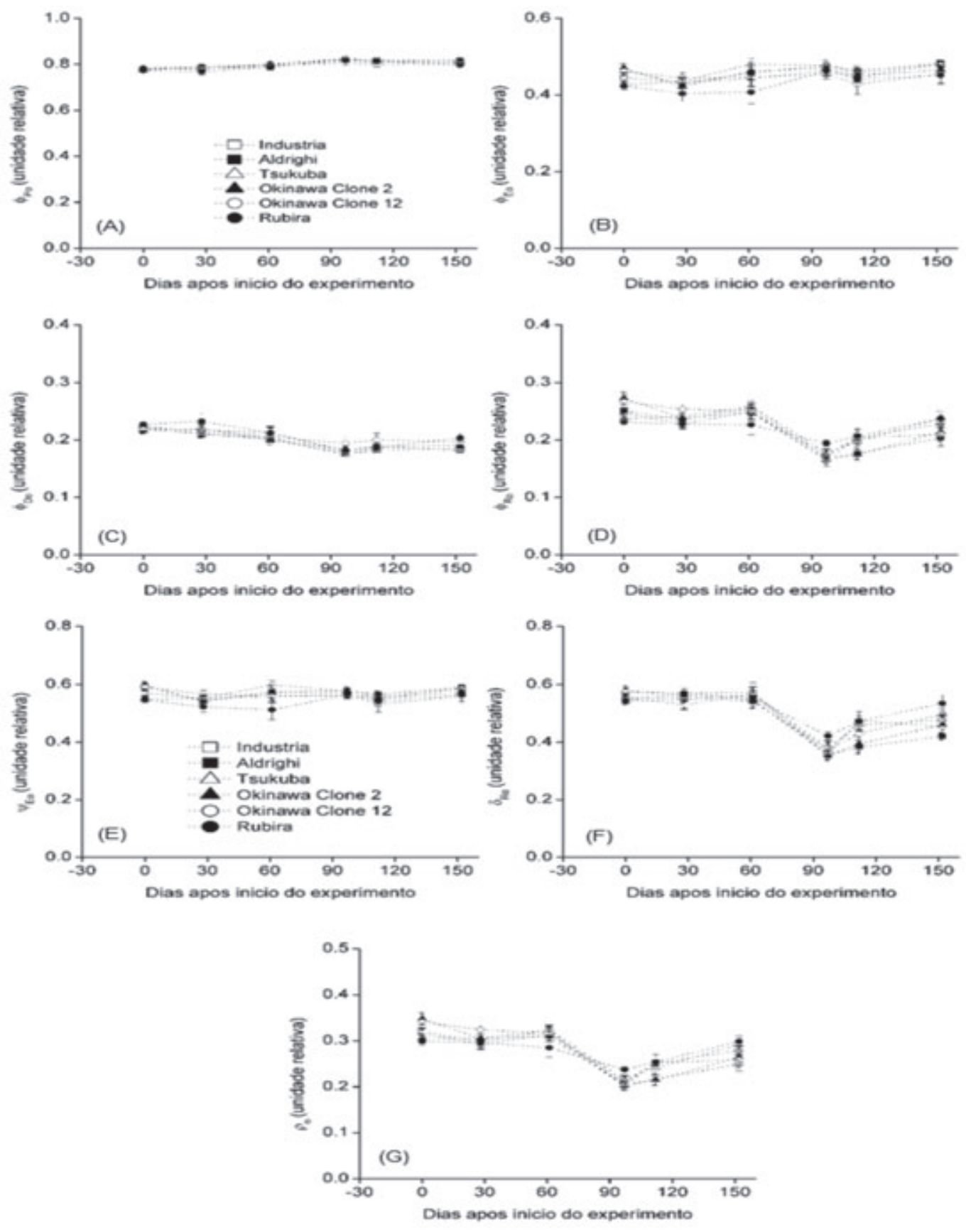

FIGURA 3- Parâmetros do Teste-JIP relacionados ao (A) rendimento quântico fotoquímico, $\Phi_{\mathrm{Po}}$; (B) Rendimento quântico do transporte de elétrons, $\Phi_{\mathrm{E}}$; (C) Rendimento quântico de dissipação de energia, $\Phi_{\mathrm{Do}}$; e (D) Rendimento quântico de transporte de elétrons além de $\mathrm{Q}_{\mathrm{A}}{ }^{-} \Phi_{\mathrm{Ro}}$; $(\mathrm{E})$ probabilidade que um éxciton capturado mova elétrons além de $\mathrm{Q}_{\mathrm{A}}-, \Psi_{\mathrm{Eo}} ;(\mathrm{F})$ eficiência com que um elétron pode ser movido do intersistema de aceptores de elétrons reduzidos para o aceptor final de elétrons do FSI $\left(\delta_{\mathrm{Ro}}\right) ;(\mathrm{G})$ eficiência com que um éxciton capturado no centro de reação pode mover um elétron dentro da cadeia de transporte de elétrons de $\mathrm{Q}_{\mathrm{A}}{ }^{-}$para os aceptores finais de elétrons do FSI $\left({ }_{\rho o}\right)$ de plantas da cultivar Maciel enxertada em seis porta-enxertos. 

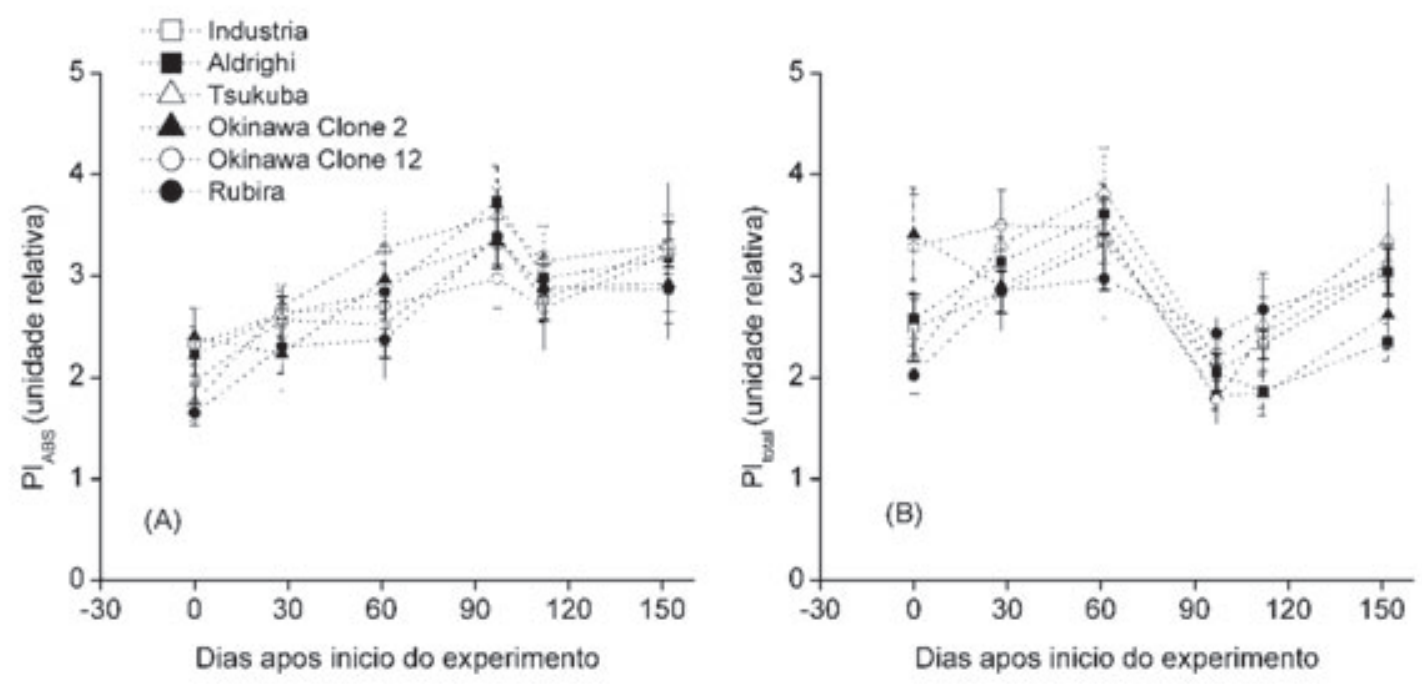

FIGURA 4- Índice de performance na base de absorção, $\mathrm{PI}_{\mathrm{ABS}}$ (A) e Índice de performance total, $\mathrm{PI}_{\mathrm{TOTAL}}$ (B) de plantas da cultivar Maciel enxertadas em seis porta-enxertos.

\section{CONCLUSÕES}

1-A combinação da cv. 'Maciel' com os porta-enxertos estudados não afeta a absorção e o aproveitamento da energia luminosa na cadeia de transporte de elétrons da fotossíntese.

2-Parâmetros de fluorescência da clorofila $a$, relacionados aos fluxos de energia por centro de reação e ao rendimento quântico de transporte de elétrons além de $\mathrm{Q}_{\mathrm{A}}$-, são mais elevados quando as folhas estão em estádio inicial de desenvolvimento.

3-Parâmetros do Teste-JIP, relacionados ao fotossistema I, são os mais afetados por alta precipitação, sendo que 'Maciel'sobre seedlings de 'Rubirá’ é a associação com menores danos ao aparato fotossintético, sob condições de alta pluviosidade.

\section{AGRADECIMENTOS}

Ao CNPq, CAPES, MCT e FAPERGS, pelo apoio financeiro.

\section{REFERÊNCIAS}

CASSOL, D; SILVA, F.S.P; FALQUETO, A.R; BACARIN, M.A. An evaluation of non-destructive methods to estimate total chlorophyll content. Photosynthetica, Prague, v. 46, p.634-636, 2008.

EMBRAPA, Boletins agroclimatológicos, Disponível em: <http://www.cpact.embrapa.br/agromet/ estacao/estacao.html>. Acesso em: 12 fev. 2010.

EULLAFFROY, P; FRANKART, C; AZIZ, A; COUDERCHET, M; BLAISE, B. Energy fluxes and driving forces for photosynthesis in Lemna minor exposed to herbicides. Aquatic Botany, Amsterdam, v. 90, p. 172-178, 2009.

GOTO, R.; SANTOS, .H.S.; CAÑIZARES, A.L. Enxertia em hortaliças. São Paulo: Editora UNESP, 2003. 75 p.

HERTER, F. G. Zoneamento agroclimático do pessegueiro e da nectarina para o Rio Grande do Sul. Pelotas: Embrapa Clima Temperado, 2002. 27 p.

HUTHER, C. M. Estresses abióticos em plantas transformadas e não transformadas de tomate 'MicroTom’ com diferentes expressões da sHSP22 mitocondrial: Efeito do alagamento e de ciclos de alta e baixa temperatura. Dissertação (Mestrado em Fisiologia Vegetal). Instituto de Biologia, Universidade Federal de Pelotas, Pelotas, 2011. 96 p. 
JIANG, C; GAO, H; ZOU, Q; JIANG G; LI, L. Leaf orientation, photorespiration and xanthophyll cycle protect young soybean leaves against high irradiance in field. Environmental and Experimental Botany, Elmsford, v. 55, p. 87-96, 2006.

PERBONI, A.T; CASSOL, D; SILVA, F.S.P; SILVA, D. M; BACARIN, M. A. Chlorophyll a fluorescence study revealing effects of flooding in canola hybrids. Biologia, Bratislava, v. 67, p. 338-346, 2012.

SCHANSKER, G; SRIVASTAVA, A; GOVINDJEE; STRASSER, R. Characterization of the $820 \mathrm{~nm}$ transmission signal paralleling the chlorophyll a fluorescence rise (OJIP) in pea leaves. Functional Plant Biology, Dordrecht, v. 30, p. 785-796, 2003.

STRASSER, B.J; STRASSER, R.J. Measuring fast fluorescence transients to address environmental questions: The JIP-test. In: MATHIS, P. (Ed.). Photosynthesis: from light to biosphere. Dordrecht: Kluwer Academic Publisher, 1995. p. 977- 980.

STRASSER, R. J.; TSIMILLI-MICHAEL, M.; SRIVASTAVA, A. Analysis of the chlorophyll a. In: PAPAGEORGIOU, G.C.; GOVINDJEE (Ed.). Fluorescence transient chlorophyll a fluorescence: a signature of photosynthesis. Netherlands: Spring, 2004. v.14, p. 321-362.
TSIMILLI-MICHAEL, M.; STRASSER, R. In vivo assessment of stress impact on plants' vitality: applications in detecting and evaluating the beneficial role of Mycorrhization on host plants. In: VARMA, A (Ed.). Mycorrhiza: state of the art, genetics and molecular biology, ecofunction, biotechnology, ecophysiology, structure and systematic. Uttar Pradesh: Springer, 2008. p. 679-703

WANG, H., WANG, F., WANG, G., MAJOURHAT, K., The responses of photosynthetic capacity, chlorophyll fluorescence and chlorophyll content of nectarine (Prunus persica var. Nectarina Maxim) to greenhouse and field grown conditions. Scientia Horticulturae, Amsterdam, v. 112, p. 66-72, 2007.

YUSUF, M.A; KUMAR, D; RAJWANSHI, R; STRASSER, R.J; TSIMILLI-MICHAEL, M; GOVINDJEE; SARIN, N.B. Overexpression of y-totopherol methyl transferase gene in transgenic Brassica juncea plants alleviates abiotic stress: Physiological and chlorophyll a fluorescence measurements. Biochimica et Biophysica Acta, Amsterdam, v. 1797, p. 1428-1438, 2010. 